\title{
Desempeño Laboral en la Gestión Municipal 2020
}

\section{RESUMEN}

José Luis Marín Cárdenas

jmarinca5@ucvvirtual.edu.pe

Escuela de posgrado

Universidad César Vallejo

ORCID: 0000-0001-9944-4979

José Manuel Delgado Bardales

jmdelgadob@ucvvirtual.edu.pe

Escuela de posgrado

Universidad César Vallejo

ORCID:0000-0001-6574-2759

Scopus autor ID: 24070333700

Código Renacyt: P0050554

La presente investigación tuvo como objetivo, Identificar las características del desempeño laboral para mejorar la gestión Municipal 2020, la investigación fue cualitativa de diseño revisión sistemática, se realizó el análisis de 10 artículos científicos relacionados a la variable de estudio. Se encontró que La evaluación de los 10 artículos científicos, enfocados al desempeño laboral en la gestión municipal pertenecen al tipo y diseño de estudios descriptivos. Los artículos enfocan al desarrollo de competencias y habilidades de los colaboradores municipales a través de capacitaciones, además se enfocan al uso de la tecnología, y $20 \%$ a metodologías aplicadas frente a los objetivos institucionales. También el desempeño laboral influye en la vida sostenible en el contexto de la crisis económica, debido a que demandan condiciones laborales diferentes, además de necesitar políticas, condiciones y consecuencias de afección pública. También se evidencia la necesidad de contar con un sistema para evaluar el desempeño laboral, por competencias, comprometidos y con comportamiento ético legal para su buen funcionamiento de los municipios y sobretodo integrado con todos los componentes del Estado. El control administrativo y financiero demanda de profesionales y técnicos con competencias integrales para el uso y disposición de recursos públicos para ser utilizados eficiente y eficazmente.

Palabras clave: Desarrollo laboral, gestión, territorio. 


\title{
Labor performance in municipal management 2020
}

\begin{abstract}
The objective of this research was to identify the characteristics of job performance to improve Municipal management 2020, the research was qualitative with a systematic review design, the analysis of 10 scientific articles related to the study variable was carried out. It was found that the evaluation of the 10 scientific articles, focused on job performance in municipal management belong to the type and design of descriptive studies. The articles focus on the development of skills and abilities of municipal collaborators through training, also focus on the use of technology, and $20 \%$ on methodologies applied against institutional objectives. Work performance also influences sustainable life in the context of the economic crisis, due to the fact that they demand different working conditions, in addition to requiring public policies, conditions and consequences. There is also evidence of the need to have a system to evaluate job performance, by competencies, committed and with legal ethical behavior for the proper functioning of the municipalities and above all integrated with all the components of the State. Administrative and financial control demands professionals and technicians with comprehensive skills for the use and provision of public resources to be used efficiently and effectively.
\end{abstract}

Keywords: Labor development, management, territory.

Artículo recibido: 03 nov. 2020

Aceptado para publicación: 07 dic. 2020

Correspondencia jmarinca5@ucvvirtual.edu.pe

Conflictos de Interés: Ninguna que declarar 


\section{INTRODUCCIÓN}

El desempeño laboral es un tema muy sensible para las organizaciones, sobre todo en aquellas de carácter público donde tienen que alcanzar los objetivos institucionales y lograr las metas propuestas por el Estado; sin embargo, muchas autoridades no le dan mayor atención por la condición política, ya que los funcionarios ejercen la función por conveniencia personal sin cumplir con las competencias determinadas en los perfiles de puestos laborales.

También debe considerarse que el desempeño laboral es pilar fundamental en el crecimiento de toda organización, porque tanto avanza, crece, se desarrolla permite responder a las condiciones y necesidades de la sociedad de lo contrario genera deficiencias que afectan el desarrollo social. Para garantizar el buen desempeño laboral de los trabajadores se debe cumplir criterios de selección y reclutamiento de los trabajadores, pero estos criterios muchas veces son obviados por factores intrínsecos de los municipios como, por ejemplo, presupuesto limitado, inestabilidad laboral a los trabajadores y ausencia de capacitaciones.

A nivel internacional, en Colombia, en un estudio realizado sobre el Modelo de Evaluación del Desempeño Basado en Competencias, indican que con una adecuada evaluación del desempeño del personal es posible identificar puntos débiles y fuertes del personal, establecer la calidad de los subordinados, reconocer el nivel de cumplimiento de las funciones administrativas, identificar la eficacia y eficiencia en el cumplimiento de funciones, definir los requisitos de un programa de selección, formular las funciones de un cargo en específico, crear la base racional para recompensar el buen desempeño y definir las capacitaciones que se realizarán. Una forma de hacerlo es evaluándolos por las competencias necesarias para llevar las tareas del cargo a cabalidad, la cual es una técnica que ha tenido gran auge en los últimos tiempos (Manjarrés, A. 2013).

La evaluación del desempeño de la gestión pública está en el centro de las preocupaciones de los tomadores de decisiones de las instituciones gubernamentales, a todos los niveles de la gestión nacional, regional y local. La exigencia por optimizar los niveles de eficiencia y eficacia en el uso de los recursos, así como generar y fortalecer los mecanismos de rendición de cuentas hacia los ciudadanos y los grupos de interés que rodean la acción pública son los fundamentos básicos que han tenido 
los gobiernos para impulsar el desarrollo de indicadores de desempeño en las instituciones públicas (Bonnefoy y Armijo. 2005).

A nivel nacional, el desempeño laboral en nuestro país viene siendo afectado por el estrés que sufren los trabajadores, por diversos problemas familiares que tienen en casa, y por la incapacidad que tienen los jefes de delegar funciones, asimismo, por la falta de identidad con la institución que representan, y se frustran muchos proyectos importantes por la incapacidad de trabajar en equipo. El $70 \%$ en el país, tanto de empresas privadas y estatales sufren de esta enfermedad, sin embargo, son pocas las firmas que adoptan una solución al respecto (Medina y Ninanya, 2019).

En el sector público existe una necesidad urgente de mejorar el desempeño laboral del personal administrativo nombrado, toda vez, que se debe salvar las reticencias de la actual cultura administrativa. Se debe contar con líderes en Recursos Humanos, debiéndose mejorar la eficiencia y organización productiva, que tiene que incidir en el reclutamiento del talento humano, que va constituir un elemento de motivación y desarrollo de habilidades y destrezas (Medina y Ninanya, 2019).

A nivel Regional, en la provincia de Moyobamba realizaron un estudio en el Ministerio público específicamente en el área de Gerencia administrativa, donde determinaron que el desempeño laboral de los trabajadores admirativos del área de Gerencia está relacionado con la modalidad de contrato (Quiroz, 2017).

En la Provincia de Juanjui en el área de la Gerencia territorial Huallaga Central Juanjui, el clima organizacional y el desempeño mantiene un nivel regular, debido a la capacidad de análisis de los colaboradores, además a las actitudes, el personal se muestra empático con las recomendaciones que el jefe realiza, no siguen con las especificaciones brindadas por el jefe inmediato sin ningún cuestionamiento, y en muchos casos el colaborador no tiene actitud positiva hacia el desarrollo de su trabajo (Naveda, 2017).

A nivel local, la Municipalidad Distrital de Huicungo presenta 45 trabajadores, entre los cuales 10 nombrados y 35 contratado, por mucho tiempo se ha observado deficiencia y bajo rendimiento en su desempeño laboral, para prueba de ello en el último plan de incentivos municipales no se recaudó lo dispuesto por las normativas del estado y a todo ello porque no se dio cumplimiento a las metas establecidas en el periodo $2014-2018$. 
Por lo expuesto se formulación el problema: ¿Cuáles son las características del desempeño laboral para mejorar la gestión Municipal 2020? Y como objetivo Identificar las características del desempeño laboral para mejorar la gestión Municipal 2020.

\section{ESTRATEGIAS METODOLÓGICAS O MATERIALES Y MÉTODOS}

El tipo de investigación es básica aplicada con revisiones sistemáticas enmarcada dentro del nivel aplicativo cuyo fin es la de resolver problemas prácticos. Es Analítica, porque en base a los resultados diagnósticos del evento a modificar y de las causas que lo originan, combinados con los elementos obtenidos en la revisión de la literatura servirá de base para desarrollar la propuesta, para el enfoque cuantitativo se utilizará el diseño descriptivo simple (Hernández, Fernández \& Baptista, 2014) Por la temporalidad es de corte transversal. Se utilizará el diseño descriptivo: se recogió información relacionada al desempeño laboral en la gestión municipal, así mismo se recogió información científica y se buscó una estrategia para solucionar el desempeño laboral a la municipalidad en estudio.

La recolección se realizar a través de una tabla de registro de datos y la fuente de revisión bibliográfica de artículos de investigación tanto internacionales como nacionales que tuvieron como tema principal el desempeño laboral para mejorar la gestión municipal, de todos los artículos buscados (10) se recogió los datos más importantes según nivel de evidencia y se descartó los menos relevantes. Se estableció la búsqueda con criterio que las características de información concuerden y discrepen en los artículos internacionales y nacionales. Además de acuerdo a criterios técnicos preestablecidos se realizó, a partir de ello, se determinó la calidad de la evidencia basa en instrumentos estandarizados, es decir un plan detallado de procedimientos que nos conduzcan a reunir datos con un propósito específico. 


\section{RESULTADOS Y DISCUSIÓN}

Resultados

Tablas 1: Estudios revisados sobre el desarrollo laboral en la gestión municipal 2020

\section{DATOS DE LA PUBLICACIÓN}

\section{Autor}

Año

Nombre de la Investigación

Alarcón Barrero, R., $\&$ Salvador Hernández, Y.
2020

La efectividad del desempeño de la gestión pública de gobiernos locales.
Revista donde se ubica la Publicación

Dilemas Contemporáneos: Educación,

Política y Valores, 7(2), 1-16. México.

http://eds.a.ebscohost.com/eds/detail/detail

?vid=7\&sid=a1263ca0-da85-41f8-bef7$8 \mathrm{a} 928 \mathrm{cb} 8 \mathrm{ac} 2 \mathrm{c} \% 40 \mathrm{sdc}-\mathrm{v}-$
Vol. 7(2), 1-16.

Volumen y Número

Mmc210Z

T11ZHMtbG12ZQ\%3d\%3d\#db=eue \&AN= 141370013

\section{CONTENIDO DE LA PUBLICACIÓN}

\begin{tabular}{cccc}
\hline $\begin{array}{c}\text { Tipo y Diseño } \\
\text { de } \\
\text { Investigación }\end{array}$ & $\begin{array}{c}\text { Población y } \\
\text { Muestra }\end{array}$ & Instrumentos & Aspectos ético \\
\hline Análisis de un & $\begin{array}{c}\text { Colaborador } \\
\text { es del } \\
\text { caso de estudio }\end{array}$ & Cuestionario & $\begin{array}{c}\text { Código de } \\
\text { ética en } \\
\text { investigación }\end{array}$ \\
\hline
\end{tabular}

\section{Resultados}

\section{Conclusión}

Se muestra el diseño y aplicación de un índice sintético que evalúa la efectividad del desempeño de gobiernos locales, resultado del estudio de propuestas metodológicas y procedimientos en sistemas empresariales $y$ gubernamentales en distintos escenarios y contextos geográficos; así como de las proyecciones de perfeccionamiento de la gestión pública local
De la formulación teórica de un conjunto de subíndices formados por indicadores relacionados con dimensiones para una gestión pública efectivas y variables definidas para la efectividad del desempeño, se realizan evaluaciones prácticas con la combinación de diferentes métodos y técnicas 
2. Autor Año Nombre de la Investigación

Ley $\mathrm{N}^{\circ} 20.922$ sobre fortalecimiento de la gestión y profesionalización del personal

Hernández

Domínguez, J. I.
2018

municipios en torno a la percepción sobre la gestión de sus recursos humanos

Revista donde se ubica la

Santiago de Chile. Revista Enfoques: Ciencia

Política y Administración Pública, 16(29), 1-

$$
25 .
$$

http://eds.a.ebscohost.com/eds/detail/detail?vid $=5 \& \operatorname{sid}=a 1263 \mathrm{ca} 0$-da85-41f8-bef7-

$$
8 \mathrm{a} 928 \mathrm{cb} 8 \mathrm{ac} 2 \mathrm{c} \% 40 \mathrm{sdc}-\mathrm{v}-
$$

sessmgr02\&bdata $=$ Jmxhbmc9ZXMmc210ZT11

ZHMtbGl2ZQ\%3d\%3d\#AN=136364842\&db=f Publicación

$16(29), 1-25$.

\section{Volumen y Número}

ua

\section{CONTENIDO DE LA PUBLICACIÓN}

\begin{tabular}{cccc}
\hline $\begin{array}{c}\text { Tipo y Diseño } \\
\text { de } \\
\text { Investigación }\end{array}$ & $\begin{array}{c}\text { Población y } \\
\text { Muestra }\end{array}$ & Instrumentos & Aspectos ético \\
\hline & & & \\
Análisis de un & $\begin{array}{c}\text { Colaborador } \\
\text { es del } \\
\text { caso de estudio }\end{array}$ & $\begin{array}{c}\text { Cuestionari } \\
\text { Municipio }\end{array}$ & $\begin{array}{c}\text { Código de ética } \\
\text { en } \\
\text { investigación }\end{array}$ \\
\hline
\end{tabular}

\section{Resultados}

La implementación de una política de recursos humanos y de un reglamento de concursos en particular, aportaría a resolver en mayor o menor medida las necesidades que ellos manifiestan al respecto. En este sentido, las preguntas que orientarán la reflexión son las siguientes: ¿Cuál es la situación de sus recursos humanos? ¿Qué percepción tienen sus encargados? ¿Qué percepción tienen otros funcionarios involucrados en su administración? ¿En qué medida la situación de sus recursos humanos limita las expectativas de mejora que promueve la ley 20.922 ? 

3. Autor
Año
Nombre de la Investigación

2020

$\&$ Cereceda
Revista donde se ubica la Publicación

Volumen y Número

Avances, 22(4), 536-551.

Havana Cuba.

Gonzales Ttito, Y. M.

Quintanilla, Y. V.
Capacitación y desempeño laboral en trabajadores de Serenazgo

http://eds.a.ebscohost.com/eds/de tail/detail? $\mathrm{vid}=15 \& \mathrm{sid}=\mathrm{a} 1263 \mathrm{ca} 0$ -da85-41f8-bef7-

$8 \mathrm{a} 928 \mathrm{cb} 8 \mathrm{ac} 2 \mathrm{c} \% 40 \mathrm{sdc}-\mathrm{v}-$ sessmgr02\&bdata $=$ Jmxhbmc9ZX Mmc210ZT11ZHMtbGl2ZQ\%3d $\% 3 \mathrm{~d} \# \mathrm{AN}=146334273 \& \mathrm{db}=$ fua

\section{CONTENIDO DE LA PUBLICACIÓN}

\begin{tabular}{|c|c|c|c|c|c|}
\hline $\begin{array}{c}\text { Tipo y Diseño } \\
\text { de } \\
\text { Investigación }\end{array}$ & $\begin{array}{c}\text { Población y } \\
\text { Muestra }\end{array}$ & Instrumentos & $\begin{array}{l}\text { Aspectos } \\
\text { ético }\end{array}$ & Resultados & Conclusión \\
\hline preexperimental & $\begin{array}{l}\text { La población } \\
\text { comprende } 48 \\
\text { trabajadores } \\
\text { asignados al } \\
\text { servicio de } \\
\text { Serenazgo } \\
\text { municipal, la } \\
\text { muestra estuvo } \\
\text { constituida por } 32 \\
\text { agentes en servicio } \\
\text { activo }\end{array}$ & $\begin{array}{c}\text { Encuesta, pretest } \\
\text { y postest }\end{array}$ & $\begin{array}{l}\text { Código } \\
\text { de ética } \\
\text { en } \\
\text { investiga } \\
\text { ción }\end{array}$ & 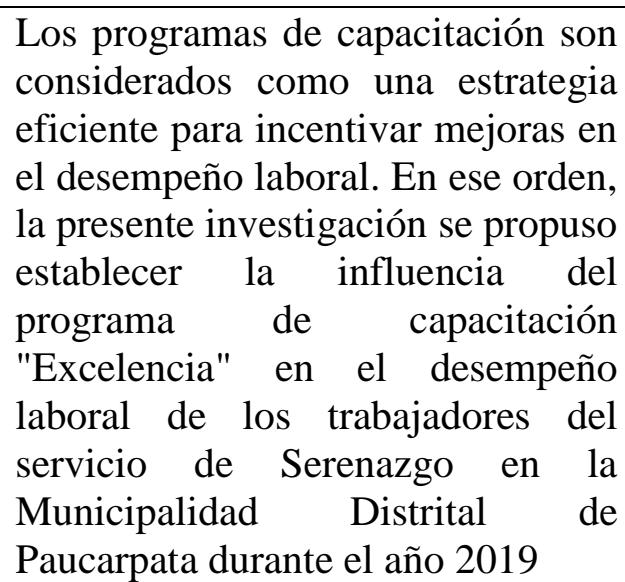 & $\begin{array}{l}\text { El programa de capacitación "Excelencia" } \\
\text { influye directa y significativamente en el } \\
\text { desempeño laboral de los trabajadores del } \\
\text { servicio de Serenazgo de la Municipalidad } \\
\text { Distrital de Paucarpata. El análisis de los } \\
\text { resultados del Pre test y Post test indican } \\
\text { que entre ambas evaluaciones hubo un } \\
\text { incremento considerable en el nivel de } \\
\text { desarrollo de las tres dimensiones del } \\
\text { desempeño laboral de los trabajadores del } \\
\text { servicio de Serenazgo }\end{array}$ \\
\hline
\end{tabular}


4. Autor Año Nombre de la Investigación

Ramos Carpio, J. P., Acosta Herrera, R. A., Ramos Carpio, K. C., \& Martínez Vásquez, Á. B
El clima organizacional y su incidencia en el desempeño laboral del Gobierno Autónomo Descentralizado Municipal del Cantón Quinsaloma, año 2018

CONTENIDO DE LA PUBLICACIÓN

\begin{tabular}{|c|c|c|c|c|c|}
\hline $\begin{array}{c}\text { Tipo y Diseño } \\
\text { de } \\
\text { Investigación }\end{array}$ & $\begin{array}{c}\text { Población y } \\
\text { Muestra }\end{array}$ & Instrumentos & $\begin{array}{l}\text { Aspectos } \\
\text { ético }\end{array}$ & Resultados & Conclusión \\
\hline $\begin{array}{c}\text { Investigación } \\
\text { cuantitativa de } \\
\text { tipo } \\
\text { descriptivocorrel } \\
\text { acional }\end{array}$ & $\begin{array}{c}\text { Colaborador } \\
\text { es } \\
\text { municipales }\end{array}$ & Cuestionario & $\begin{array}{c}\text { Código de } \\
\text { ética en } \\
\text { investigación }\end{array}$ & $\begin{array}{l}\text { Se evidenció que el clima } \\
\text { organizacional } \\
\text { directamente en el desempeño } \\
\text { laboral de los colaboradores, y } \\
\text { consecuentemente, } \\
\text { funcionario tiene un mejor } \\
\text { desempeño }\end{array}$ & $\begin{array}{l}\text { Cuando percibe un mejor clima; esto se comprobó } \\
\text { mediante la prueba estadística del chi cuadrado; } \\
\text { aceptando la hipótesis alternativa y rechazando la } \\
\text { nula, con lo cual se propuso un plan de mejora } \\
\text { orientado a fortalecer el desempeño laboral entre } \\
\text { los funcionarios }\end{array}$ \\
\hline
\end{tabular}




\section{Autor}

Corrêa, I., Camões,

M., Meyer-Sahling, J., Mikkelsen, K., \&

Distorções de incentivo ao desempenho e redução de motivação no serviço público federal no Brasil
Revista donde se ubica la Publicación

Revista Do Serviço Público

(Civil Service Review), 71(3), 297-329. Brasil.

https://doi.org/10.21874/rsp.v71i

\section{CONTENIDO DE LA PUBLICACIÓN}

\begin{tabular}{|c|c|c|c|c|c|}
\hline \multicolumn{6}{|c|}{ CONTENIDO DE LA PUBLICACIÓN } \\
\hline $\begin{array}{l}\text { Tipo y Diseño d } \\
\text { Investigación }\end{array}$ & $\begin{array}{r}\text { Població } \\
\text { Muestı }\end{array}$ & Instrun & entos & Resultados & Conclusión \\
\hline $\begin{array}{l}\text { Investigación } \\
\text { cuantitativa de } \\
\text { tipo descriptivo }\end{array}$ & $\begin{array}{l}\text { Colaborador } \\
\text { es } \\
\text { municipales }\end{array}$ & $\begin{array}{l}\text { Cuestionari } \\
\mathrm{o}\end{array}$ & $\begin{array}{ll}\text { Código de } \\
\text { ética en } \\
\text { investigació } \\
n\end{array}$ & $\begin{array}{ll}\text { En la estructura } & \mathrm{L} \\
\text { actual de carreras } & \mathrm{p} \\
\text { en el gobierno } & \mathrm{q} \\
\text { federal brasileño, } & \mathrm{E} \\
\text { los funcionarios } & \text { te } \\
\text { públicos que no } & \mathrm{d} \\
\text { disfrutan un puesto } & \mathrm{m} \\
\text { de DAS tienen dos } & \mathrm{cc} \\
\text { formas } & \mathrm{ft} \\
\text { predominantes de } & \mathrm{re} \\
\text { alcanzar un nivel de in } & \text { la } \\
\text { salario más alto } & \text { la } \\
& \mathrm{p}\end{array}$ & $\begin{array}{l}\text { La antigüedad y la aprobación en examen para una carrera en el servicio } \\
\text { público que tenga una mejor remuneración. Proponemos en este trabajo } \\
\text { que estas vías afectan negativamente la motivación en el servicio público. } \\
\text { Esas alternativas transfieren los incentivos de los funcionarios de } \\
\text { temprana carrera del desempeño laboral a la preparación para exámenes } \\
\text { de carrera que ofrecen mejores salarios, así como privan aquellos con } \\
\text { mayor antigüedad en su carrera de oportunidades de ascenso. También } \\
\text { causan inequidad salarial entre las carreras, desmotivando a los } \\
\text { uncionarios con responsabilidades similares en las carreras de más baja } \\
\text { remuneración. Datos de una encuesta con } 2.800 \text { servidores públicos en } 14 \\
\text { instituciones del gobierno federal proporcionan evidencia sugestiva para } \\
\text { la importancia de reducir las distorsiones de los incentivos en el servicio } \\
\text { público }\end{array}$ \\
\hline
\end{tabular}




\section{CONTENIDO DE LA PUBLICACIÓN}

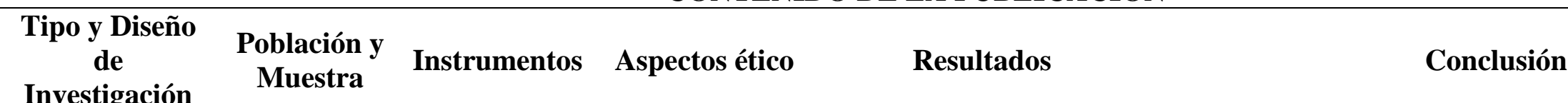

Investigación

cuantitativo de

investigación

transversal
403

Colaborador

es

municipales
Encuesta

Cuestionari

$\mathrm{O}$
Aunque los investigadores han descubierto muchas de las consecuencias beneficiosas y positivas del compromiso laboral, poco se sabe acerca de la multitud de factores antecedentes que conducen a la participación de los empleados, como la personalidad. Propósito de la investigación: El objetivo del estudio era investigar la relación entre los cinco grandes rasgos de personalidad y el compromiso laboral entre los trabajadores municipales. Motivación para el estudio: La motivación de este estudio es examinar la relación entre la personalidad y las condiciones psicológicas. Se basaba en investigaciones anteriores que la personalidad está asociada con el comportamiento de muchos empleados
La apertura a la experiencia, la conciencia, la extraversión y la agradableidad tenían una relación positiva con el compromiso laboral, mientras que el neuroticismo tiene una relación negativa con el compromiso laboral. Los municipios y las cualificaciones educativas tuvieron un impacto en la contratación laboral. Implicaciones prácticas/gerenciales: El estudio recomienda a los gerentes que pasen de un enfoque basado en la intervención a un enfoque basado en la selección, ya que los municipios pueden maximizar sus recursos al ser capaces de predecir mejor el éxito laboral al principio del proceso de selección en lugar de tratar de maximizar el rendimiento de forma continua a través de intervenciones. Contribución/valor añadido: Este estudio se suma a una comprensión de las influencias de la personalidad en los resultados laborales, como la contratación laboral, dando áreas para la exploración en el coaching o entrevistas de retroalimentación basadas en la evaluación de la personalidad. 


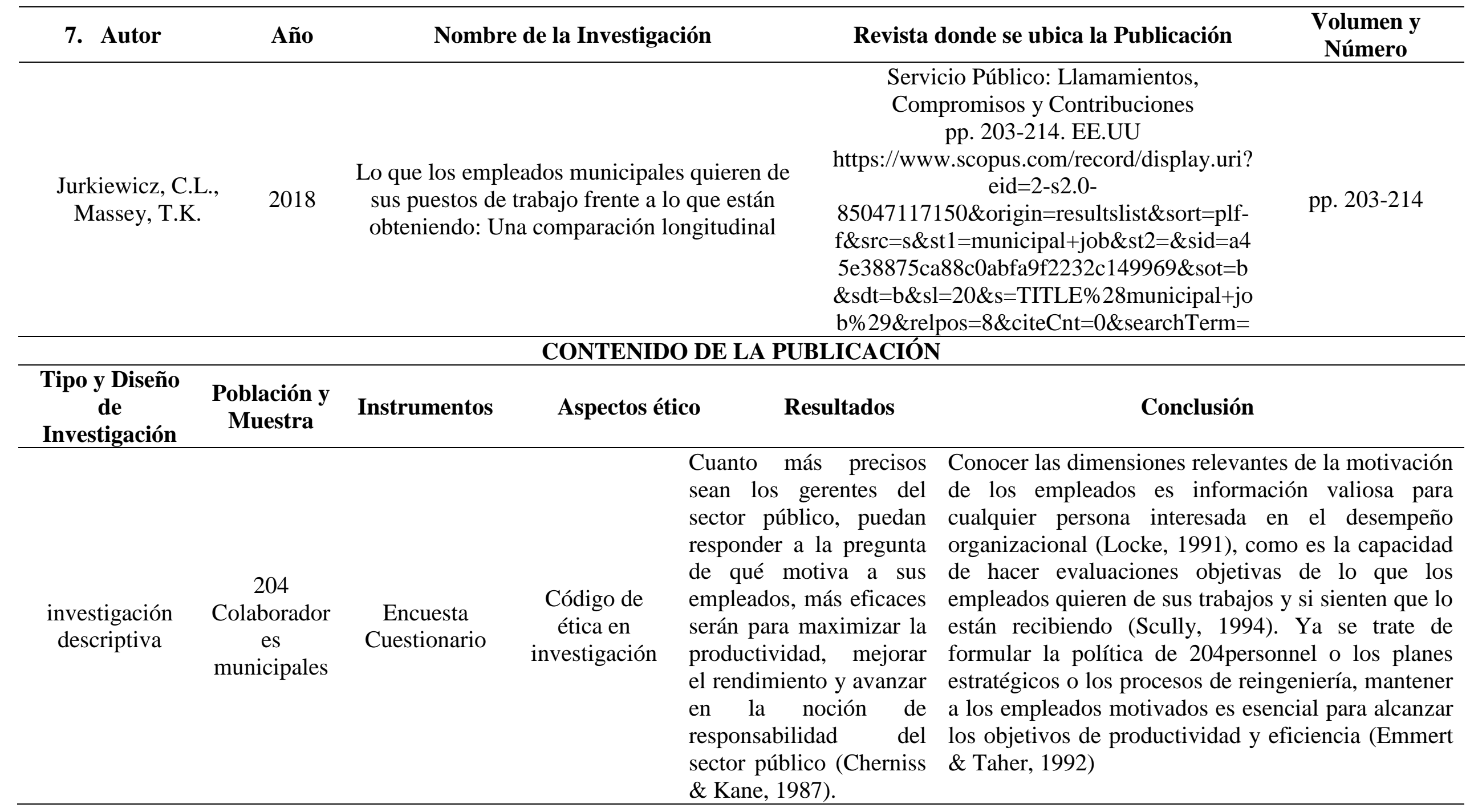




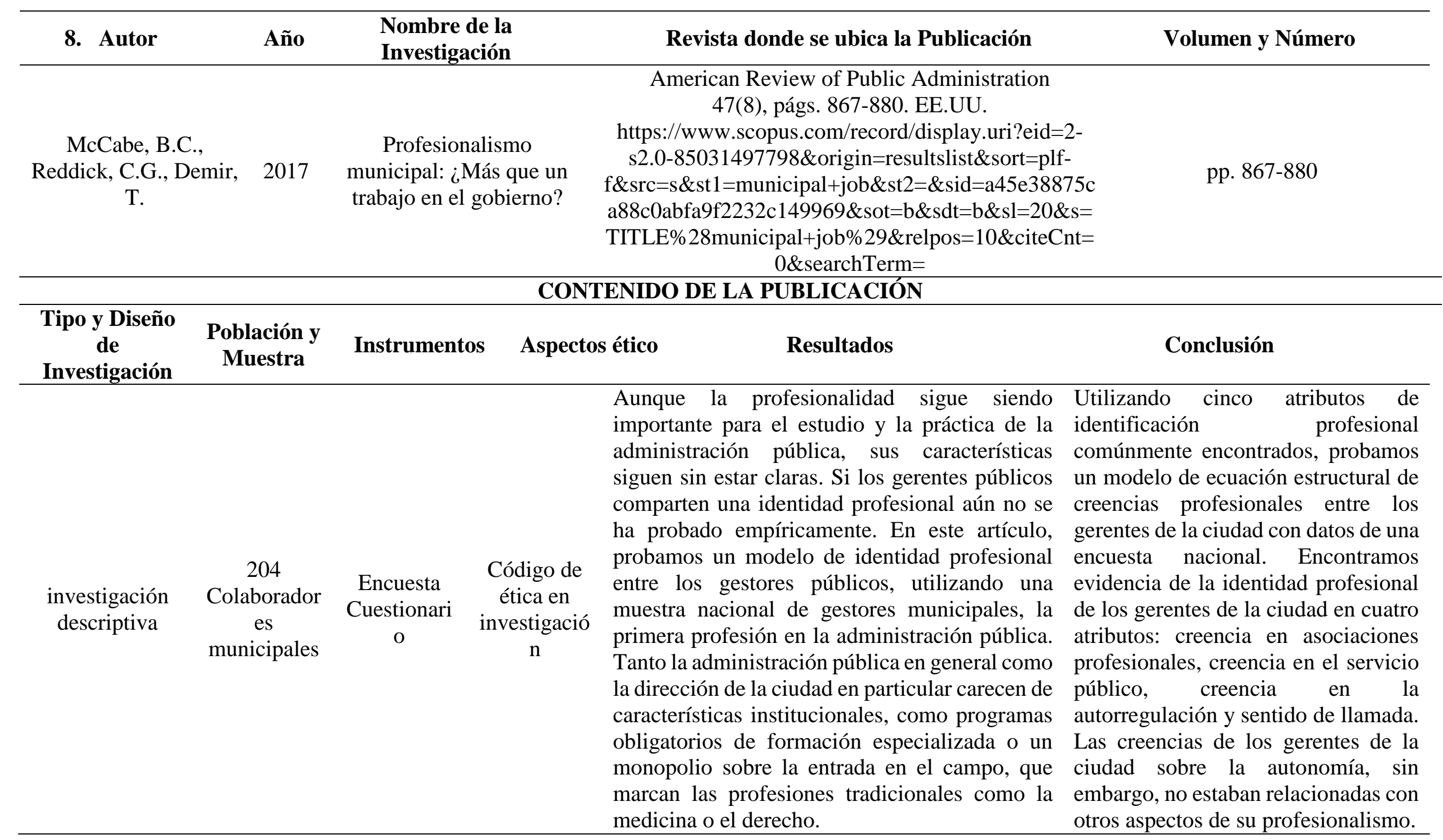




\section{Autor}

Nombre de la Investigación

Prakash, K.C.,

Neupane, S., Leino-

Arjas, P., (...),

Ilmarinen, J., Nygard, C.-H.
Perfiles laborales y discapacidades en la vida posterior: un seguimiento de 28 años de los empleados municipales en Finlandia
Revista donde se ubica la Publicación

Archivos Internacionales de Salud

Ocupacional y Ambiental

89(6), págs. 997-1007. Finlandia

https://www.scopus.com/record/display.uri

$$
\text { ?eid=2-s2.0- }
$$

$84965032383 \&$ origin $=$ resultslist\&sort $=$ plf -

$\mathrm{f} \& \mathrm{src}=\mathrm{s} \& \mathrm{st} 1=$ municipal + job $\& \mathrm{st} 2=\& \mathrm{sid}=\mathrm{a}$ 45 e38875ca88c0abfa9f2232c149969\&sot= b\&sdt=b\&sl=20\&s=TITLE\%28municipal + job $\% 29 \&$ relpos $=13 \&$ citeCnt $=7 \&$ searchT
Volumen y Número

erm $=$

\section{CONTENIDO DE LA PUBLICACIÓN}

\begin{tabular}{|c|c|c|c|c|c|}
\hline \\
\hline $\begin{array}{c}\text { Tipo y Diseño } \\
\text { de } \\
\text { Investigación }\end{array}$ & $\begin{array}{c}\text { Población y } \\
\text { Muestra }\end{array}$ & Instrumentos & Aspectos & Resultados & Conclusión \\
\hline $\begin{array}{c}\text { investigación } \\
\text { descriptiva }\end{array}$ & $\begin{array}{c}2998 \\
\text { empleados } \\
\text { municipales } \\
(1892 \\
\text { mujeres y } \\
1106 \\
\text { hombres) de } \\
\text { entre } 44 \text { y } \\
58 \text { años al } \\
\text { inicio }\end{array}$ & $\begin{array}{l}\text { ntrevistas y } \\
\text { observacion } \\
\text { es en las } \\
\text { plazas de } \\
\text { trabajo al } \\
\text { inicio }\end{array}$ & $\begin{array}{c}\text { Código de } \\
\text { ética en } \\
\text { investigació } \\
n\end{array}$ & $\begin{array}{l}\text { Después de ajustar la edad, la } \\
\text { socioeconómica, el estilo de vida y las } \\
\text { características relacionadas con la salud, las } \\
\text { mujeres en las organizaciones auxiliares (RR } \\
\text { 2.1,95\% CI 1.4-3.2), atención domiciliaria } \\
(2.1,1.4-3.2) \text {, supervisión de la cocina (2.0, } \\
\text { 1.1-3.6) y oficina (1.6, 1.1-2.4) los perfiles } \\
\text { de trabajo tenían un mayor riesgo de } \\
\text { discapacidad en la vida más tarde que los de } \\
\text { los trabajos administrativos. Los perfiles de } \\
\text { trabajo auxiliares (1.5, 1.1-2.9) y de } \\
\text { supervisión técnica }(1.7,1.1-2.7) \text { conllevan } \\
\text { un mayor riesgo entre los hombres. }\end{array}$ & $\begin{array}{l}\text { Los perfiles de trabajo de mediana edad } \\
\text { relacionados principalmente con el trabajo } \\
\text { físicamente pesado fueron fuertes } \\
\text { predictores de discapacidad en la vida } \\
\text { posterior. En las mujeres, el trabajo de } \\
\text { oficina también aumentó el riesgo de } \\
\text { discapacidad }\end{array}$ \\
\hline
\end{tabular}




\begin{tabular}{|c|c|c|c|c|c|}
\hline 10. Autor & Año & \multicolumn{2}{|c|}{ Nombre de la Investigación } & $\begin{array}{c}\text { Revista donde se ubica la } \\
\text { Publicación }\end{array}$ & Volumen y Número \\
\hline $\begin{array}{c}\text { Silva E, Silva G, } \\
\text { Bautista J. }\end{array}$ & 2017 & \multicolumn{2}{|c|}{$\begin{array}{c}\text { Influencia del clima organizacional en el desempeño } \\
\text { laboral de los trabajadores de la municipalidad de } \\
\text { morales, Región San Martín }\end{array}$} & $\begin{array}{lc}\text { acional en el desempeño } & \text { Rev. Tzhoecoen 2018 VOL. 10/ No 1, } \\
\text { de la municipalidad de } & \text { ISSN: 1997-8731. Chiclayo Perú. } \\
\text { http://revistas.uss.edu.pe/index.php/tzh } \\
\text { San Martín }\end{array}$ & $\begin{array}{l}\text { VOL. } 10 \text { / N No } 1 \text {, } \\
\text { ISSN: } 1997-8731\end{array}$ \\
\hline \multicolumn{6}{|c|}{ CONTENIDO DE LA PUBLICACIÓN } \\
\hline $\begin{array}{l}\text { Tipo y Diseño } \\
\text { de } \\
\text { Investigación }\end{array}$ & $\begin{array}{c}\text { Población y } \\
\text { Muestra }\end{array}$ & $\begin{array}{c}\text { Instrument } \\
\text { oS }\end{array}$ & $\begin{array}{l}\text { Aspectos } \\
\text { ético }\end{array}$ & Resultados & \\
\hline $\begin{array}{c}\text { Investigación } \\
\text { descriptiva } \\
\text { Correlacional y } \\
\text { método } \\
\text { inductivo }\end{array}$ & $\begin{array}{l}75 \\
\text { colaborador } \\
\quad \text { es }\end{array}$ & $\begin{array}{c}\text { Encuesta } \\
\text { cuestionario }\end{array}$ & $\begin{array}{l}\text { Código de } \\
\text { ética en } \\
\text { investigació } \\
\text { n }\end{array}$ & 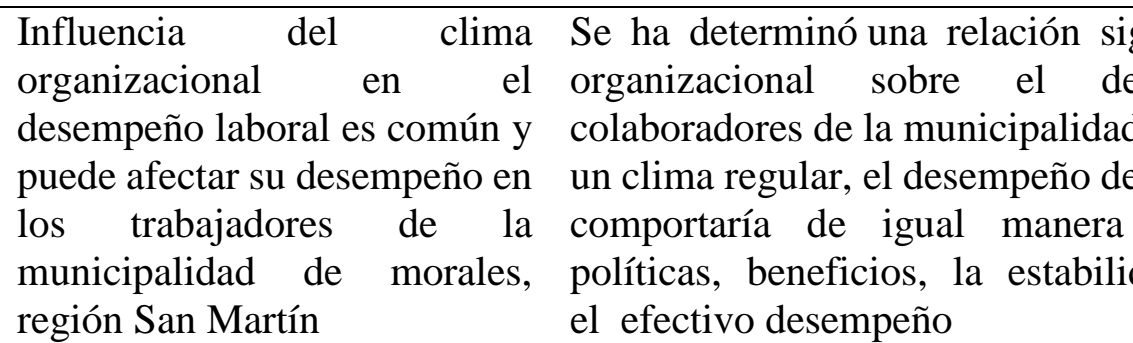 & $\begin{array}{l}\text { gnificativa del clima } \\
\text { sempeño de los } \\
\text { l, ya que, al mantener } \\
\text { los colaboradores se } \\
\text { debido a que las } \\
\text { dad laboral, impiden }\end{array}$ \\
\hline
\end{tabular}




\section{DISCUSIÓN}

Posterior a la revisión sistemática de los artículos, el $80 \%$ corresponden de a América latina y El Caribe, resaltando los países de Perú, México y Estados Unidos de América con $20 \%$ respectivamente, seguido de los países de Chile, Cuba, Brasil, Perú con el 10\%; y el $20 \%$ corresponden a los continentes de Europa y África con los países de Finlandia y Sudáfrica con 105 respectivamente.

En relación a la variable desempeño laboral en el municipio, Alarcón Barrero, R., \& Salvador Hernández, Y., determinó que, el diseño y aplicación de un índice sintético que evalúa la efectividad del desempeño de gobiernos locales. formulación teórica de un conjunto de subíndices formados por indicadores relacionados con dimensiones para una gestión pública efectivas y variables definidas para la efectividad del desempeño. Además, Hernández Domínguez, J. I., quien establece que, la implementación de una política de recursos humanos y de un reglamento de concursos en particular, aportaría a resolver en mayor o menor medida las necesidades que ellos manifiestan al respecto. Igualmente, Gonzales Ttito, Y. M., \& Cereceda Quintanilla, Y. V., concluye que, El programa de capacitación "Excelencia" influye directa y significativamente en el desempeño laboral de los trabajadores del servicio. Por otro lado, Ramos J, Acosta R, Ramos K, \& Martínez Á, determinaron que, el clima laboral interviene en los colaboradores, y a consecuencia negativa puede tener menor desempeño.

Desde el punto de vista de los resultados de los artículos analizados se puede concluir adicionalmente que los líderes de talento humano perciben un menor impacto generado por los cambios, al implementar planes de capacitación y desarrollo de competencias y que as su vez, contribuye a mejorar o a reducir la tensión en el clima organizacional por efecto de los cambios y transformaciones organizacionales, reduciendo la incertidumbre, disminuyendo el efecto del rumor y mejorando la participación de los equipos y sus miembros como factor de protección o favorecedor del cambio. Esto a su vez, confirma que reduce las tenciones que se expresan en la teoría de Lewin (1951) sobre resistencia al cambio.

También, Corrêa, I., Camões, M., Meyer-Sahling, J., Mikkelsen, K., \& Schuster, C., encontraron que, los funcionarios públicos que no disfrutan un puesto de DAS tienen dos 
formas predominantes de alcanzar un nivel de salario más alto, hecho que salen los valores institucionales y de desempeño de los colaboradores

Por su parte, Mhlanga, T, Mjoli, T., Chamisa, S. concluyen que, la experiencia, la conciencia, la extraversión y la condición agradable tenían una relación positiva con el compromiso laboral, mientras que el neuroticismo tiene una relación negativa con el compromiso laboral. Condiciones que complican el desempeño laboral. Igualmente, Jurkiewicz, C, Massey, T. encontraron que, conocer las dimensiones relevantes de la motivación de los empleados es información valiosa para cualquier persona interesada en el desempeño organizacional. Asimismo, McCabe, B, Reddick, C., Demir, T., concluyen que, utilizando cinco atributos de identificación profesional comúnmente encontrados, probamos un modelo de ecuación estructural de creencias profesionales entre los gerentes de la ciudad con datos de una encuesta nacional. Prakash, K., Neupane, S., Leino-Arjas, P., Ilmarinen, J., Nygard, C., establecen que, los perfiles de trabajo de mediana edad relacionados principalmente con el trabajo físicamente pesado fueron fuertes predictores de discapacidad en la vida posterior. En las mujeres, el trabajo de oficina también aumenta el riesgo de discapacidad. También, Silva E, Silva G, Bautista J., concluye que, una relación significativa del clima organizacional sobre el desempeño de los colaboradores de la municipalidad, al ser el clima organizacional regular el desempeño laboral sería igual.

Desde otro punto de vista, los líderes de talento humano consideran que los procesos de formación hacen a sus colaboradores más susceptibles de ser reclutados por la competencia, esta cifra parece coincidir con el rango de empresas que contemplan dentro de sus indicadores aquellos relacionados con el impacto de la cualificación del talento humano en los resultados organizacionales y su relación con los índices de rotación por efecto de la competencia y mejores niveles económicos, contratación y estabilidad. Es importante tener en cuenta que resulta necesario revisar los esquemas de gestión de conocimiento que realizan las empresas analizadas, ya que aun cuando existe un impacto positivo sobre el desempeño a corto y largo plazo de los colaboradores; sin embargo se desaprovecha potencial al dejar de lado la sensibilización de los procesos para desarrollar competencias previas al cambio, así como la socialización y la transmisión de conocimientos y la medición permanente de del impacto de la retroalimentación de la capacitación sobre los resultados del cambio organizacional. 


\section{CONCLUSIÓN O CONSIDERACIONES FINALES}

La evaluación de los 10 artículos científicos, enfocados al desempeño laboral en la gestión municipal pertenecen al tipo y diseño de estudios descriptivos. El 50\% de los artículos científicos se encuentran enfocados al desarrollo de competencias y habilidades de los trabajadores municipales a través de capacitaciones, $30 \%$ se encuentra enfocado al uso de la TIC, para lograr la gestión y $20 \%$ menciona el uso de la coeficiencia y metodologías aplicadas a al mapeo de objetivos institucionales.

El $70 \%$ de los estudios relacionados con el desempeño laboral influyen en la vida sostenible en el contexto de la crisis económica, debido a que demandan políticas, condiciones y consecuencias de afección pública.

Es importante contar con un sistema para evaluar el desempeño laboral, así como contar con colaboradores responsables, competencias, comprometidos y con comportamiento ético legal para su buen funcionamiento de los municipios y sobretodo integrado con todos los componentes del Estado.

El control administrativo y financiero demanda de profesionales y técnicos con competencias integrales para el uso y disposición de los diferentes recursos públicos para ser utilizados eficiente y eficazmente.

El clima laboral influye en el desempeño de los colaboradores, por ende, demanda generar compromisos de conlleven al cambio e identidad institucional.

\section{LISTA DE REFERENCIAS}

Alarcón Barrero, R., \& Salvador Hernández, Y. 2020 La efectividad del desempeño de la gestión pública de gobiernos locales. Dilemas Contemporáneos: Educación, Política y Valores, 7(2), 1-16. México. http://eds.a.ebscohost.com/eds/detail/detail?vid=7\&sid=a1263ca0-da85-41f8-bef7$8 \mathrm{a} 928 \mathrm{cb} 8 \mathrm{ac} 2 \mathrm{c} \% 40 \mathrm{sdc}-\mathrm{v}-$ sessmgr02\&bdata=Jmxhbmc9ZXMmc210ZT11ZHMtbG12ZQ\%3d\%3d\#db=eue\&A $\mathrm{N}=141370013$

Bonnefoy J, Armijo M. (2005). Indicadores de desempeño en el sector público. Instituto Latinoamericano y del Caribe de Planificación Económica y Social - ILPES. Publicación de las Naciones Unidas. ISSN impreso 1680-886X. ISSN electrónico 1680-8878. ISBN: 92-1-322800-7. 
https://www.cepal.org/ilpes/noticias/paginas/0/26120/manual45.pdf

Corrêa, I., Camões, M., Meyer-Sahling, J., Mikkelsen, K., \& Schuster, C. (2020). Distorções de incentivo ao desempenho e redução de motivação no serviço público federal no Brasil. Revista Do Serviço Público (Civil Service Review), 71(3), 297329. https://doi.org/10.21874/rsp.v71i3.3408

Gonzales Ttito, Y. M., \& Cereceda Quintanilla, Y. V. (2020). Capacitación y desempeño laboral en trabajadores de Serenazgo. Avances, 22(4), 536-551. Havana Cuba. http://eds.a.ebscohost.com/eds/detail/detail?vid=15\&sid=a1263ca0-da85-41f8bef7-8a928cb8ac2c\%40sdc-vsessmgr02\&bdata=Jmxhbmc9ZXMmc210ZT11ZHMtbG12ZQ\%3d\%3d\#AN=14633 $4273 \& d b=$ fua

Hernández Domínguez, J. I. (2018). Ley n²0.922 sobre fortalecimiento de la gestión y profesionalización del personal municipal: un estudio de caso en dos municipios en torno a la percepción sobre la gestión de sus recursos humanos. revista enfoques: ciencia política y administración pública, 16(29), 1-25. http://eds.a.ebscohost.com/eds/detail/detail?vid=5\&sid=a1263ca0-da85-41f8-bef7$8 \mathrm{a} 928 \mathrm{cb} 8 \mathrm{ac} 2 \mathrm{c} \% 40 \mathrm{sdc}-\mathrm{v}-$ sessmgr02\&bdata=jmxhbmc9zxmmc210zt1lzhmtbgl2zq\%3d\%3d\#an=136364842\& $\mathrm{db}=$ fua

Hernández, Fernández y Baptista (2016). Metodología de la Investigación. Sexta edición, México. Recuperado de: http://observatorio.epacartagena.gov.co/wpcontent/uploads/2017/08/metodologia-de-la-investigación-sextaedicion.compressed.pdf

Jurkiewicz, C.L., Massey, T.K. 2018 Lo que los empleados municipales quieren de sus puestos de trabajo frente a lo que están obteniendo: Una comparación longitudinal Servicio Público: Llamamientos, Compromisos y Contribucionespp. 203-214. EE.UU. https://www.scopus.com/record/display.uri?eid=2-s2.0$85047117150 \&$ origin $=$ resultslist $\&$ sort $=$ plf $\mathrm{f} \& \mathrm{src}=\mathrm{s} \& \mathrm{st} 1=$ municipal+job\&st $2=\& \mathrm{sid}=\mathrm{a} 45 \mathrm{e} 38875 \mathrm{ca} 88 \mathrm{c} 0 \mathrm{abfa} 9 \mathrm{f} 2232 \mathrm{c} 149969 \& \mathrm{~s}$ $\mathrm{ot}=\mathrm{b} \& \mathrm{sdt}=\mathrm{b} \& \mathrm{sl}=20 \& \mathrm{~s}=\mathrm{TITLE} \% 28$ municipal + job$\% 29 \&$ relpos $=8 \&$ citeCnt $=0 \&$ sear 
chTerm= pp. $203-214$

Manjarrés A., Castell R., Luna C. (2013). INGENIARE, Universidad Libre-Barranquilla, Año 8, No. 15, pp. 11-29 • ISSN: 1909-2458. Modelo de Evaluación del Desempeño Basado en Competencias. https://dialnet.unirioja.es/descarga/articulo/5980476.pdf

McCabe, B.C., Reddick, C.G., Demir, T. 2017 Profesionalismo municipal: ¿Más que un trabajo en el gobierno? American Review of Public Administration. 47(8), págs. 867-880. EE.UU. https://www.scopus.com/record/display.uri?eid=2-s2.085031497798\&origin=resultslist\&sort=plf-

$\mathrm{f} \& \mathrm{src}=\mathrm{s} \& \mathrm{st} 1=$ municipal+job\&st $2=\& \mathrm{sid}=\mathrm{a} 45 \mathrm{e} 38875 \mathrm{ca} 88 \mathrm{c} 0 \mathrm{abfa} 9 \mathrm{f} 2232 \mathrm{c} 149969 \& \mathrm{~s}$ $\mathrm{ot}=\mathrm{b} \& \mathrm{sdt}=\mathrm{b} \& \mathrm{sl}=20 \& \mathrm{~s}=\mathrm{TITLE} \% 28$ municipal + job $\% 29 \&$ relpos $=10 \& \mathrm{citeCnt}=0 \& \mathrm{se}$ $\operatorname{archTerm}=\quad$ pp. $867-880$

Medina C., Ninanya E. (2019). La gestión de talento humano y el desempeño docente en dos instituciones educativas de Lima metropolitana. Tesis de posgrado. Universidad Marcelino Champagnat. Escuela de postgrado. Programa de maestría Perú. http://repositorio.umch.edu.pe/bitstream/UMCH/3017/1/82.\%20Tesis\%20\%28Med ina $\% 20 \% 20 y \% 20$ Ninanya\%29.pdf

Mhlanga, T.S., Mjoli, T.Q., Chamisa, S.F. 2019 Personalidad y compromiso laboral entre los trabajadores municipales de la provincia oriental del Cabo, Sudáfrica SA Journal of Human Resource Management. 17,a1188. Cabo Oriental, Sudáfrica. $\quad$ https://www.scopus.com/record/display.uri?eid=2-s2.0$85075058053 \&$ origin $=$ resultslist $\&$ sort $=$ plf-

$\mathrm{f} \& \mathrm{src}=\mathrm{s} \& \mathrm{st} 1=$ municipal+job\&st $2=\& \operatorname{sid}=\mathrm{a} 45 \mathrm{e} 38875 \mathrm{ca} 88 \mathrm{c} 0 \mathrm{abfa} 9 \mathrm{f} 2232 \mathrm{c} 149969 \& \mathrm{~s}$ $\mathrm{ot}=\mathrm{b} \& \mathrm{sdt}=\mathrm{b} \& \mathrm{sl}=20 \& \mathrm{~s}=\mathrm{TITLE} \% 28$ municipal + job $\% 29 \&$ relpos=3\&citeCnt $=1 \&$ sear chTerm

Naveda K (2017). Clima organizacional y su influencia en el desempeño laboral de los trabajadores del Proyecto Especial Alto Huallaga. Tesis de pregrado. Universidad de Huánuco. Facultad de Ciencias Empresariales. Programa académico profesional de administración de empresas. http://repositorio.udh.edu.pe/bitstream/handle/123456789/344/T047_47461367T.p df? sequence $=1 \&$ is Allowed $=\mathrm{y}$ 
Prakash, K.C., Neupane, S., Leino-Arjas, P., (...), Ilmarinen, J., Nygard, C.-H. 2016

Perfiles laborales y discapacidades en la vida posterior: un seguimiento de 28 años de los empleados municipales en Finlandia Archivos Internacionales de Salud Ocupacional y Ambiental 89(6), págs. 997-1007. Finlandia https://www.scopus.com/record/display.uri?eid=2-s2.0$84965032383 \&$ origin=resultslist\&sort=plf$\mathrm{f} \& \mathrm{src}=\mathrm{s} \& \mathrm{st} 1=$ municipal + job $\& \mathrm{st} 2=\& \mathrm{sid}=\mathrm{a} 45 \mathrm{e} 38875 \mathrm{ca} 88 \mathrm{c} 0 \mathrm{abfa} 9 \mathrm{f} 2232 \mathrm{c} 149969 \& \mathrm{~s}$ $\mathrm{ot}=\mathrm{b} \& \mathrm{sdt}=\mathrm{b} \& \mathrm{sl}=20 \& \mathrm{~s}=\mathrm{TITLE} \% 28$ municipal + job $\% 29 \&$ relpos $=13 \& \mathrm{citeCnt}=7 \& \mathrm{se}$ $\operatorname{archTerm}=$

Quiroz P. (2017). Gestión del potencial humano y su relación con el desempeño laboral de los trabajadores de la Gerencia Administrativa del Ministerio Público de San Martin, distrito de Moyobamba. Tesis de maestría. San Martín Perú. http://repositorio.ucv.edu.pe/bitstream/handle/20.500.12692/31627/quiroz_cp.pdf? sequence $=1 \&$ isAllowed $=\mathrm{y}$

Ramos Carpio, J. P., Acosta Herrera, R. A., Ramos Carpio, K. C., \& Martínez Vásquez, Á. B. (2019). El clima organizacional y su incidencia en el desempeño laboral del Gobierno Autónomo Descentralizado Municipal del Cantón Quinsaloma, año 2018. (Spanish). Dilemas Contemporáneos: Educación, Política y Valores, 7, 1-32.Toluca México. http://eds.a.ebscohost.com/eds/detail/detail?vid=17\&sid=a1263ca0-da8541f8-bef7-8a928cb8ac2c\%40sdc-v sessmgr02\&bdata=Jmxhbmc9ZXMmc210ZT11ZHMtbG12ZQ\%3d\%3d\#AN=13957 $2023 \& d b=$ eue

Silva E, Silva G, Bautista J. 2017 Influencia del clima organizacional en el desempeño laboral de los trabajadores de la municipalidad de morales, Región San Martín Rev. Tzhoecoen 2018 VOL. 10 / No 1, ISSN: 1997-8731. Chiclayo Perú. http://revistas.uss.edu.pe/index.php/tzh/article/view/781/pdf

Tamayo y Tamayo (2003). El proceso de la investigación científica. Cuarta edición. México. Editorial Limusa SA. ISBN 968-18-5872-75.4. https://books.google.com.mx/books?id=BhymmEqkkJwC\&printsec=frontcover\& $\mathrm{hl}=\mathrm{es} \mathrm{v}=$ onepage $\& \mathrm{q} \& \mathrm{f}=\mathrm{false}$ 